\title{
Active Control and Closed-Loop Identification of Flutter Instability in Typical Section Airfoil
}

\author{
Mark A. McEver* \\ L-3 Communications Corporation, Pittsburgh, Pennsylvania 15238 \\ Emil V. Ardelean $\ddagger$ \\ Science Applications International Corporation, Albuquerque, New Mexico 87106 \\ Daniel G. Cole \\ University of Pittsburgh, Pittsburgh, Pennsylvania 15237 \\ and \\ Robert L. Clark토 \\ Duke University, Durham, North Carolina 27708
}

DOI: $\underline{10.2514 / 1.26335}$

\begin{abstract}
This research used the technique of $Q$ parameterization to identify an unstable model of an airfoil above its flutter boundary. A nominal, stabilizing controller was designed and implemented on a typical section airfoil with an articulated trailing-edge flap. Although stabilized by the nominal controller, the open-loop, unstable plant was identified from closed-loop signals at increasing flow speeds. The plant model identified at the highest flow speed was then used to design a new controller using the Evans root-locus technique. In a series of wind tunnel tests, the nominal controller increased the flutter boundary by $30 \%$ above the open-loop flutter speed, and the redesigned controller increased the boundary by $52 \%$.
\end{abstract}

\section{Nomenclature}

$A=$ state transition matrix

$B \quad=$ input matrix

$C=$ output matrix

$D=$ feedthrough matrix

$d \quad=$ disturbance

$G \quad=$ actual plant

$G_{0}=$ nominal plant model

$H=$ disturbance shaping filter

$j=\sqrt{-1}$

$K_{1}=$ nominal feedback controller

$K_{2}=$ redesigned feedback controller

$M_{0}=$ left coprime factor of $G_{0}$

$N_{0}=$ left coprime factor of $G_{0}$

$r=$ reference input

$s=$ Laplace transform variable

$U_{\infty}=$ flow speed

$u=$ plant input

$X=$ left coprime factor of $K$

$Y=$ left coprime factor of $K$

$y \quad=$ plant output

$\alpha=$ input of $\Delta$

$\beta=$ output of $\Delta$

$\Delta=$ plant $Q$ parameter

$\lambda=$ plant pole

$\zeta=$ damping ratio of pole

Presented as Paper 1973 at the 45th AIAA/ASME/ASCE/AHS/ASC Structures, Structural Dynamics and Materials Conference, Palm Springs, CA, 19-22 April 2004; received 5 July 2006; revision received 3 November 2006; accepted for publication 1 December 2006. Copyright () 2007 by the American Institute of Aeronautics and Astronautics, Inc. All rights reserved. Copies of this paper may be made for personal or internal use, on condition that the copier pay the $\$ 10.00$ per-copy fee to the Copyright Clearance Center, Inc., 222 Rosewood Drive, Danvers, MA 01923; include the code 0731-5090/ $07 \$ 10.00$ in correspondence with the CCC.

${ }^{*}$ Control Systems Engineer, 615 Epsilon Drive. Member AIAA.

${ }^{\dagger}$ Senior Mechanical Engineer, 2109 Air Park Road SE. Member AIAA.

${ }^{\ddagger}$ Assistant Professor, 648 Benedum Hall, 3700 O’Hara Street. Member AIAA.

${ }^{\S}$ Professor, Box 90300. Member AIAA.

$$
\begin{array}{ll}
\omega & =\text { radian frequency } \\
\omega_{n} & =\text { natural frequency of pole }
\end{array}
$$

\section{Introduction}

$\mathbf{F}$ LUTTER is an aeroelastic phenomenon that occurs when airflow couples energy into airfoil structural vibration modes, causing dynamic instability and destructive vibration [1]. This type of fluid-structure interaction occurs at and above a flow speed known as the critical speed or flutter speed. In general, the flutter speed of an airfoil or wing depends on properties of both the fluid and the structure, which together form a coupled aeroelastic system. There have been considerable efforts to delay the onset of flutter in particularly susceptible aircraft, such as those with high-aspect-ratio wings [2-4]. Toward this end, researchers have suppressed flutter in wind tunnel tests by articulating trailing-edge flaps with a closedloop control system [5-7]. However, because the dynamics of the aeroelastic system change significantly with flow, fixed-parameter controllers may only stabilize the flutter mode over a small range of flow speed within the aircraft flight envelope, and multiple, different controllers may be needed to cover the entire design flight regime. Adaptive control has been studied as a means to compensate for timevarying dynamics of aeroelastic systems [8].

System identification techniques can measure the actual flutter behavior and unstable dynamics of aeroelastic systems [9]. In many instances these identified models can supplement or enhance analytical flutter prediction and modeling. Because the aeroelastic plant is open loop unstable above its flutter speed, any system identification technique for measuring the unstable dynamics must use signals measured on the stabilized, closed-loop system. Using an adaptive control technique involving $Q$ parameterization of the identified model structure, this article presents a method by which the unstable plant, stabilized by an arbitrary feedback controller, can be represented as a function of an unknown, stable system. This unknown, stable system can be easily identified from closed-loop signals.

This work describes a method of designing flutter controllers based on the identified, $Q$-parameterized models of the unstable aeroelastic system at supercritical flow speeds. An initial nominal controller is designed that stabilizes an airfoil over a limited range of speeds above the open-loop flutter speed, permitting identification of 


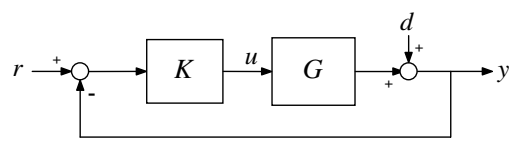

Fig. 1 Negative feedback control system.

the $Q$-parameterized model. Each successive model of the unstable system is then used to design a new controller for that specific range of flow speeds. This entire model identification/controller design sequence can proceed over the entire range of operational flow speeds, and could prove useful for aircraft that are desired to have large flight envelopes, such as unmanned aerial vehicles (UAVs) and gossamer aircraft. Although this work deals with a single-input/ single-output (SISO) closed-loop aeroelastic system, the extension of the $Q$-parameterization technique to multiple-input/multipleoutput (MIMO) systems is straightforward. As will be shown in the sequel, the $Q$ parameterization uses a coprime factorization of the system. The system theory for MIMO coprime factorization exists and its application for MIMO flutter control is apparent [10].

\section{Closed-Loop Identification Using $Q$ Parameterization}

Real-time system identification is one method of measuring variations in plant dynamics, and many computational techniques exist for creating various plant models from experimental input/ output data [11]. For identifying plants in an open-loop setting, these algorithms are well defined and give satisfactory results. However, an unstable aeroelastic system above flutter can only be identified in a stable closed loop. Plant identification from closed-loop measurements can lead to biased estimates and a general identifiability problem [11].

Consider the open-loop system identification problem by assuming for the moment that the controller $K(s)$ in Fig. 1 is disconnected from the plant. Many identification techniques exist for estimating $G$ from measurements of $u$ and $y$ including spectral analysis, subspace methods, and instrument variable approach [11]. A common technique is the stochastic spectral method that solves for the complex frequency response function $G(\omega)$ according to

$$
\Phi_{y u}(\omega)=G(\omega) \Phi_{u u}(\omega)+\Phi_{d u}(\omega)
$$

where $\Phi$ is a power spectrum. Because $u$ and $d$ are independent processes, $\Phi_{d u}=0$, and $G(\omega)$ can be solved from

$$
G(\omega)=\Phi_{y u}(\omega) \Phi_{u u}^{-1}(\omega)
$$

In the closed-loop environment, the input to the plant $u$ is a filtered version of $y+d$, making $\Phi_{d u} \neq 0$. Open-loop system identification techniques will give erroneous and biased estimates of $G$. One remedy is to estimate the closed-loop system $P(s)=G(s)$ / $[1+G(s) K(s)]$ from measurements of $y$ and a persistently exciting reference signal $r$. The plant can then be calculated from the relationship

$$
G(s)=\frac{P(s)}{1-P(s) K(s)}
$$

It is not always necessary to identify plant dynamics over the entire control bandwidth, but rather to update or augment the model in way that is relevant to the controller. $Q$ parameterization performs this function by providing a framework for describing uncertainty between the actual plant and nominal model as it pertains to closedloop behavior, and a method by which to estimate this uncertainty from closed-loop signals.

To overcome the difficulties of closed-loop identification, Hansen, Tay, and Schrama [12-14] independently developed an idea based on $Q$ parameterization of the plant model. This involves describing the plant as a function of two components: 1) a known, nominal plant transfer function and 2) a transfer function parameter, called the $Q$ parameter, representing the difference between the true plant and the nominal plant. Assume the nominal plant $G_{0}$ is modeled as the ratio of two stable systems, $G_{0}=N_{0} M_{0}^{-1}$, and a stable, stabilizing controller for the plant is also described as the ratio of two stable systems $K=X Y^{-1}$. If the functions $N_{0}, M_{0}, X$, and $Y$, known as coprime factors, are chosen such that the Bezout identity $M_{0} Y-$ $N_{0} X=1$ is satisfied, then the set of all plants stabilized by $K$ can be parameterized according to [12-14]

$$
G=\frac{N_{0}+Y \Delta}{M_{0}+X \Delta}
$$

From the block diagram in Fig. 2, it can be shown [12] that the equations for the input and output of $\Delta, \alpha$, and $\beta$, can be written as

$$
\begin{aligned}
\alpha & =X r \\
\beta & =M_{0} y-N_{0} u \\
& =\Delta \alpha+\left(M_{0}+\Delta X\right) H d
\end{aligned}
$$

Given an unknown, potentially unstable plant stabilized by some controller $K$, one can choose functions $X, Y, N_{0}$, and $M_{0}$, estimate $\Delta$ from measurements of $r, u$, and $y$ (see Fig. 3 ), and then calculate the estimate of $G$ from Eq. (4). Notice that estimating $\Delta$ from $\alpha$ and $\beta$ is an open-loop identification problem, because $r$ is statistically uncorrelated with the disturbance $d$.

This technique is useful in identifying unstable aeroelastic systems for two reasons. First, the parameter $\Delta$ is always stable, even if $G$ is unstable. Second, identifying $\Delta$ from closed-loop signals is possible using any one of the multitude of open-loop system identification techniques. A method for implementing the technique is proposed as follows.

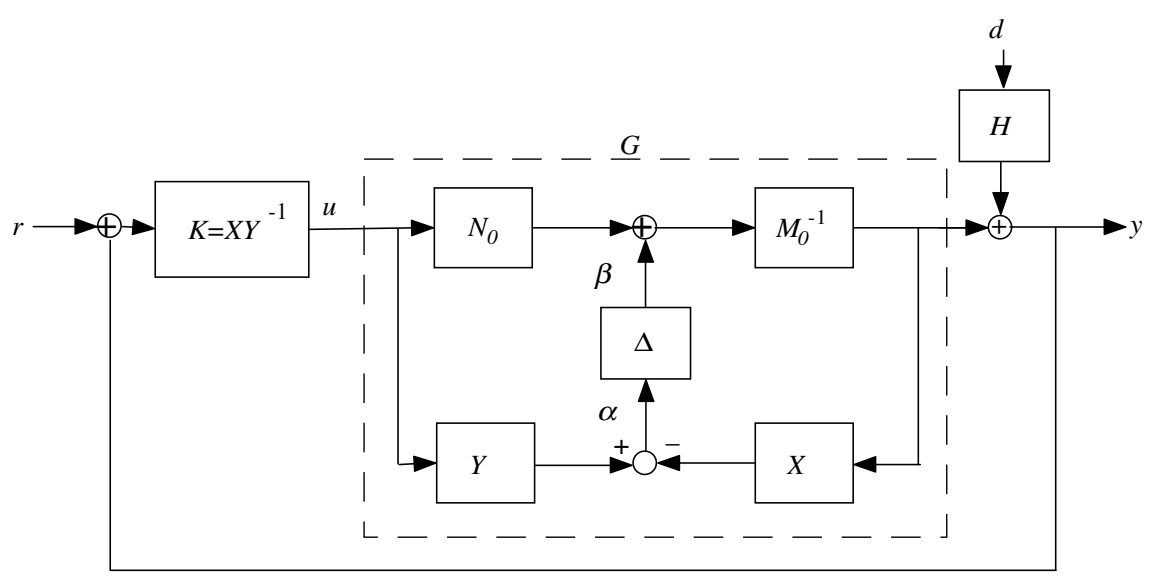

Fig. $2 Q$ parameterization of plant model. 


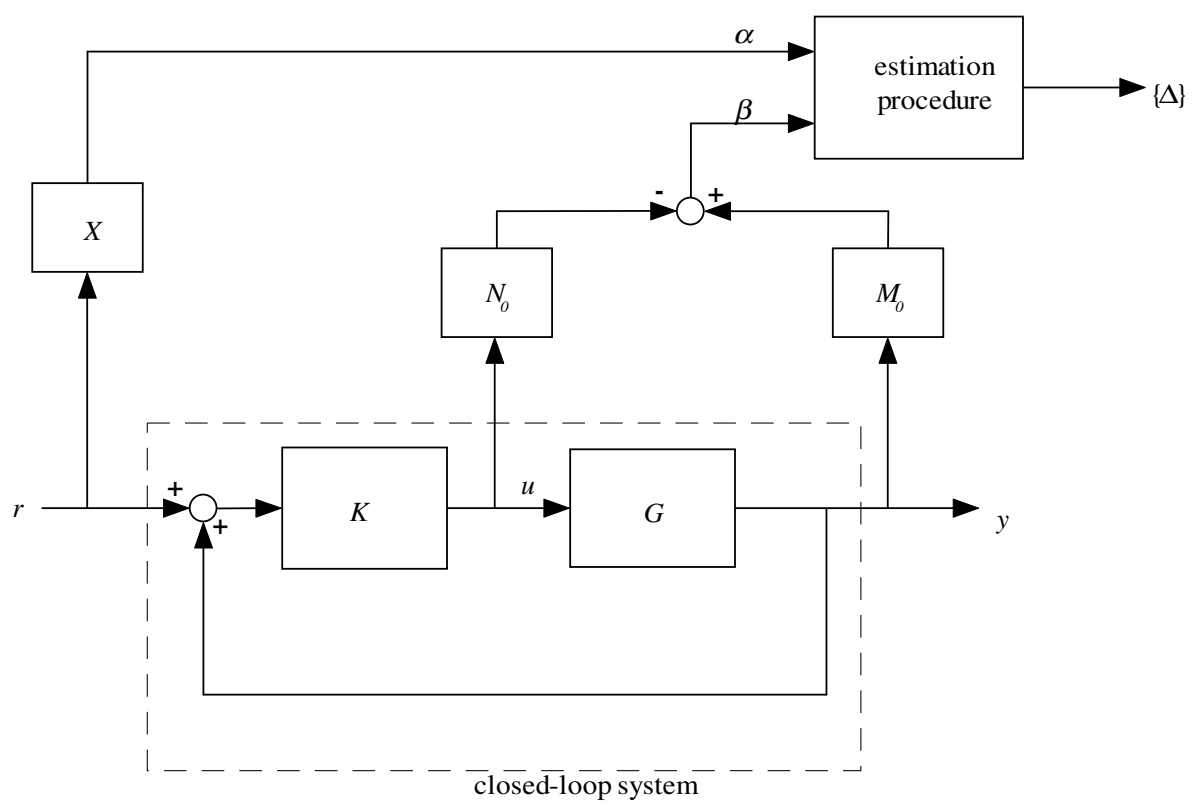

Fig. 3 Closed-loop identification of $\Delta$.

1) Model the stable aeroelastic system at a flow speed just below flutter analytically or from experimental data. This model is designated as the nominal plant.

2) Design a feedback controller to stabilize the wing for some range of flow speeds above the open-loop flutter speed.

3 ) Using the nominal plant model and controller, find coprime factors $N_{0}, M_{0}, X$, and $Y$

4) Identify $\Delta$ from measurements on the closed-loop system and compute a model of the unstable plant from Eq. (4)

This procedure can repeat at flow speeds above the open-loop flutter boundary, but still within the stabilizing range of the controller. The models of the unstable aeroelastic system at higher flow speeds within this range will be more useful for predicting stability margins of the closed-loop system and will allow the controller to be redesigned to better stabilize the system at higher flow speeds. In theory, this controller design/plant identification process could be performed sequentially at increasing flow speeds, resulting in a set of controllers that stabilize the system over a continuous range of flow speeds above the open-loop flutter speed. With sufficient controller overlap, an adaptive flutter control scheme could switch controllers according to flow speed. This process would yield lower order controllers than sequentially closing multiple, nested feedback loops around each other.

\section{Flutter Control Test Bed}

A NACA 0015 airfoil with a trailing-edge flap suitable for flutter testing was designed and constructed [7]. The internal frame consists of balsa wood, aluminum, and steel and is covered in an outer skin of 0.01 in.-thick aluminum sheet. Overall dimensions of the airfoil are $0.52 \mathrm{~m}$ span and $0.36 \mathrm{~m}$ chord. A piezoelectric actuator housed in the middle of the airfoil actuates the flap. The actuator, known as a Vstack actuator [15], consists of two piezoelectric stacks placed in a steel frame and energized such that one stack contracts while the other expands, causing a small differential motion. This motion is transferred to the flap via a slider-crank lever mechanism and generates $0.044 \mathrm{deg}$ flap deflection per actuator volt. The flap achieves a maximum deflection of $\pm 5.5 \mathrm{deg}$ at the maximum allowable voltage of $\pm 125 \mathrm{~V}$.

Flutter testing is performed in the subsonic wind tunnel at Duke University. The airfoil is situated in the $0.70 \times 0.51 \times 1.22 \mathrm{~m}$ test section on a rig permitting pitch and plunge degrees of freedom. Angular displacement sensors mounted directly at the pivot points measure flap angle and pitch angle, while plunge displacement is measured with an angular displacement sensor mounted at the end of a thin rod that rotates as the airfoil plunges. At zero flow condition, the pitch and plunge natural frequencies were measured as 5.9 and $3.1 \mathrm{~Hz}$, respectively. Tests show the onset of coupled pitch-plunge flutter occurs at a freestream velocity $U_{\infty}=23 \mathrm{~m} / \mathrm{s}$, and at a frequency of $4.3 \mathrm{~Hz}$.

Figure 4 shows the setup used in the wind tunnel experiments. A dSPACE digital signal processor (DSP) board computes control commands at a rate of $500 \mathrm{~Hz}$ while a SigLab signal analyzer collects data. A flap servo control system was designed to track flap deflection commands with a bandwidth of $10 \mathrm{~Hz}$. The servo controller consists of proportional-integral control and a notch filter that compensates for a flap/actuator mode at $30 \mathrm{~Hz}$. Airflow has little or no effect on the servo control performance due to the high force output of the $\mathrm{V}$-stack actuator. Because the servo controller achieves good tracking performance, the dynamics from commanded flap deflection to pitch and plunge are dominated by airfoil dynamics and not by actuator/ flap dynamics. The flutter controller commands flap deflection, rather than actuator voltage.

During testing, particularly at $U_{\infty} \geq 10 \mathrm{~m} / \mathrm{s}$, nonuniform flow and wind tunnel effects buffet the airfoil, causing small movements in pitch and plunge. This ambient "noise," although small in magnitude, is significant when compared to the motion generated by the white noise source articulating the flap during system identification. It became necessary to use the swept-sine feature of the SigLab signal analyzer to yield a satisfactory signal-to-noise ratio. The SigLab analyzer commands a 2 deg amplitude sinusoidal flap deflection at frequencies slowly varied from 1 to $6 \mathrm{~Hz}$. At subcritical flow speeds, this technique is used to estimate frequency responses from the commanded flap angle to pitch angle and plunge displacement. At supercritical flow speeds, with the nominal controller stabilizing the airfoil, this technique is used to estimate the frequency response of $\Delta$ from the filtered signals in Fig. 3. Frequency-domain system identification software then fits continuous-time state-space models to the estimated frequency responses. Open-loop and closed-loop damping ratios are obtained from these models.

Suppressing 1 degree of freedom effectively stabilizes the airfoil, because the airfoil motions are coupled. With this in mind, a SISO control strategy was developed based on feeding the backpitch angle. Pitch was chosen as an appropriate measure of the airfoil state due to its high interaction with the airflow, as well as the higher coupling between flap deflection and pitch than flap deflection and plunge.

After some design iteration, it became clear that feeding backpitch through a second-order controller was sufficient to stabilize the airfoil. This simple strategy is known in the structural control field as 


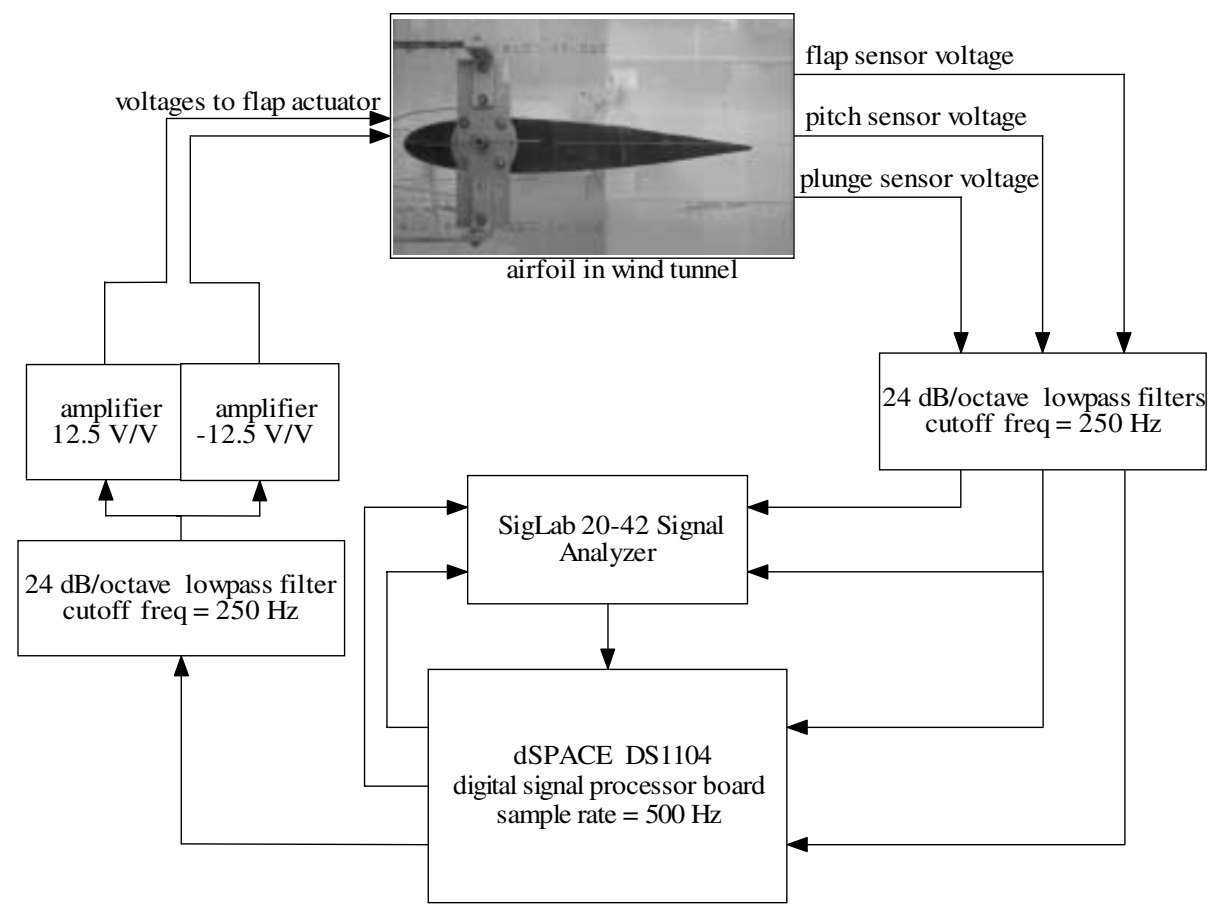

Fig. 4 Flutter control experimental setup.

positive position feedback (PPF) and is conceptually similar to a tuned vibration damper [16]. The nominal controller design yielded a second-order filter of the form

$$
K_{1}(s)=\frac{g_{1} \omega_{1}^{2}}{s^{2}+2 \zeta_{1} \omega_{1} s+\omega_{1}^{2}}
$$

with a gain $g_{1}=-0.13$, damping ratio $\zeta_{1}=0.1$, and natural frequency $\omega_{1}=28.3(4.5 \mathrm{~Hz})$. The negative gain is necessary due to the $180 \mathrm{deg}$ phase shift between actuator voltage and pitch sensor voltage. The nominal controller increased the flutter boundary $30 \%$, from 23 to $30 \mathrm{~m} / \mathrm{s}$. Figure 5 shows the open-loop and closed-loop time responses of the pitch angle at $U_{\infty}=24 \mathrm{~m} / \mathrm{s}$.

\section{Closed-Loop Identification Results}

Identifying the unstable, open-loop plant from measurements of the nominal closed-loop system was the focus of the wind tunnel experiments. This involved choosing coprime factors $N_{0}, M_{0}, X$, and $Y$ so that the plant parameter $\Delta$ could be identified from the filtered signals $\alpha$ and $\beta$. Figure 6 shows the flutter control architecture in the program computed by the DSP. $K_{1}$ is the nominal flutter controller transformed to discrete time with a Tustin transformation, and $K_{2}$ is the controller redesigned for higher flow speeds.

Any choice of coprime factors is valid provided the Bezout identity $M_{0} Y-N_{0} X=1$ is satisfied. The method employed in this work is based on a technique outlined in Feedback Control Theory by Doyle et al. [17]. First, we must design the nominal plant $G_{0}$ and choose its coprime factors $N_{0}$ and $M_{0}$. Any plant model that closes a stable feedback loop with the nominal controller is valid. Therefore,

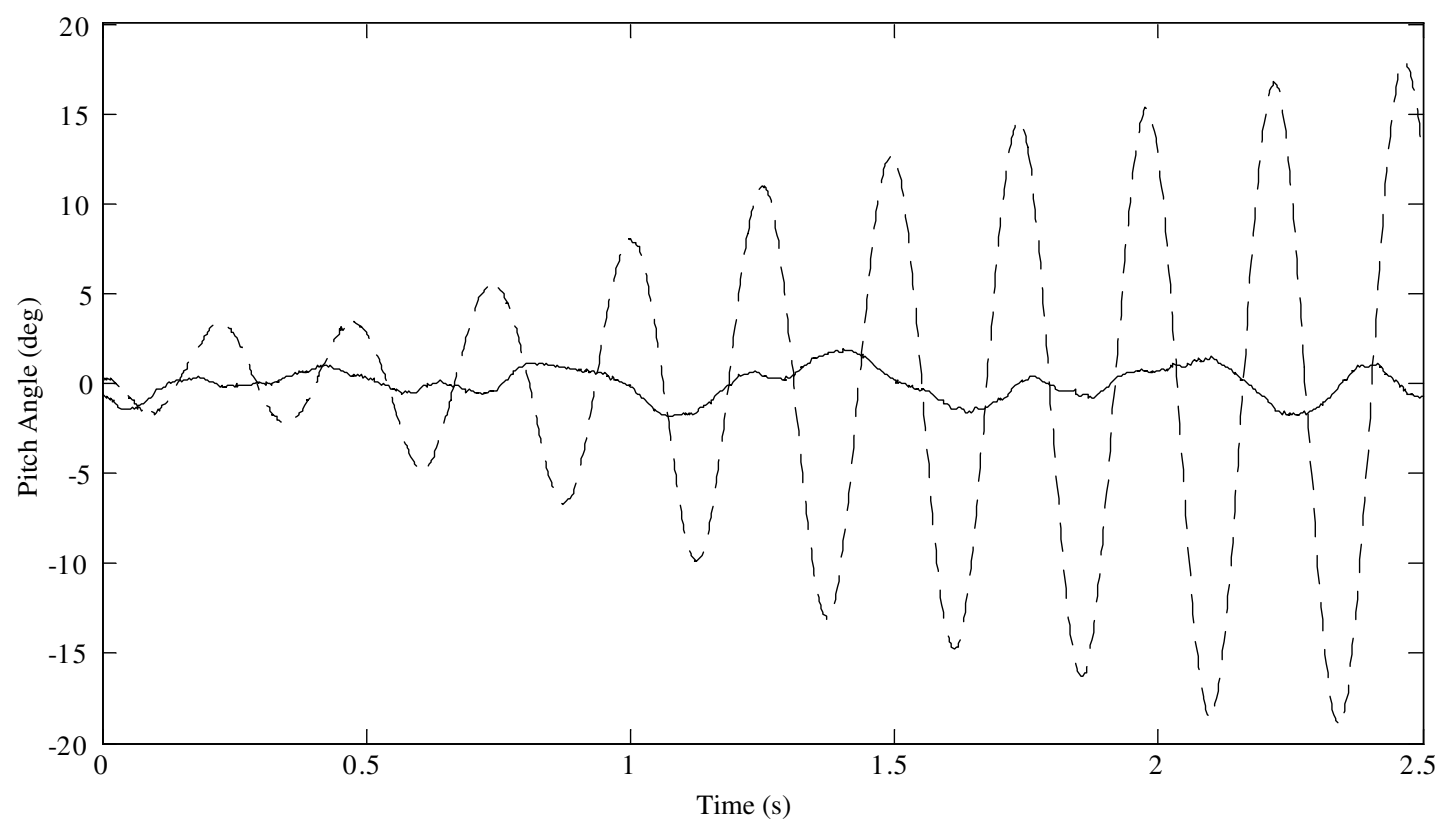

Fig. 5 Pitch angle at $U_{\infty}=24 \mathrm{~m} / \mathrm{s}$, open loop (dashed) and closed loop (solid) with $K_{1}$. 


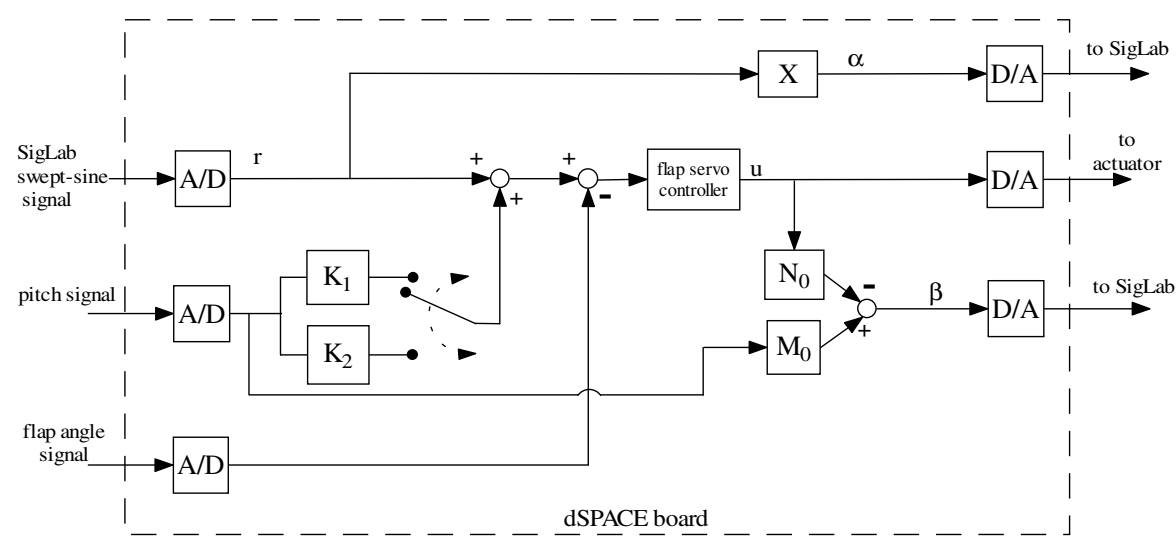

Fig. 6 Flutter controller and system identification architecture.

we choose $G_{0}$ to be a first-order low-pass filter with unity gain and a $6 \mathrm{~Hz}$ cutoff frequency. This simple design is motivated by a desire to use low order coprime factors, and the fact that a significant difference between the nominal plant and the actual plant makes $\Delta$ more identifiable [10]. Defining a state-space description of $G_{0}$

$$
G_{0}(s) \stackrel{s}{=}\left[\begin{array}{ll}
A & B \\
C & D
\end{array}\right]
$$

coprime factors can be computed from

$$
\begin{gathered}
N_{0}(s) \stackrel{s}{=}\left[\begin{array}{cc}
A+B F & B \\
F & 1
\end{array}\right] \\
M_{0}(s) \stackrel{s}{=}\left[\begin{array}{cc}
A+B F & B \\
C+D F & D
\end{array}\right]
\end{gathered}
$$

where $F$ is chosen such that the eigenvalues of $A+B F$ are stable. The next step is to find $X$ and $Y$ such that $X Y^{-1}=K_{1}$ and $M_{0} Y-N_{0} X=1$. Define initial coprime factors of the continuoustime nominal controller $K_{1}(s)$ as

$$
\begin{gathered}
\bar{X}(s)=-\frac{\operatorname{num}\left\{K_{1}(s)\right\}}{\Pi(s)} \\
\bar{Y}(s)=\frac{\operatorname{den}\left\{K_{1}(s)\right\}}{\Pi(s)}
\end{gathered}
$$

where num \{\} and $\operatorname{den}\{\}$ are the polynomial numerator and denominator, respectively, and $\Pi(s)$ is a stable, second-order polynomial necessary to make $\bar{X}(s)$ and $\bar{Y}(s)$ proper. The coefficients of $\Pi(s)$ are typically chosen to make its poles higher in frequency than the expected plant dynamics. Let

$$
v(s)=N_{0}(s) \bar{X}(s)+M_{0}(s) \bar{Y}(s)
$$

then compute

$$
\begin{gathered}
X(s)=-\bar{X}(s) v^{-1}(s) \\
Y(s)=\bar{Y}(s) v^{-1}(s)
\end{gathered}
$$

The systems $N_{0}, M_{0}, X$, and $Y$ now form the basis for the $Q$ parameterized plant model and can be used to estimate $\Delta$.

To test the accuracy of the $Q$-parameterized system identification technique, a model of the open-loop plant was estimated from a $\Delta$ identified in closed-loop below the flutter boundary, at $U_{\infty}=$ $22.5 \mathrm{~m} / \mathrm{s}$. The frequency response of flap angle input to pitch angle output was calculated from this model and compared to a measured open-loop frequency response obtained directly from the input and output. Figure 7 shows both frequency responses. The frequency response magnitudes matched very well, to within a few decibels, and the phases generally agreed over the bandwidth of interest except for a difference of $35 \mathrm{deg}$ at $4.2 \mathrm{~Hz}$. This phase difference may indicate a problem of numerical sensitivity introduced into the estimation process, possibly by the choice of coprime factors. For the purposes of the experiments, this error was deemed small enough to not significantly compromise the usefulness of the technique in estimating an unstable plant from closed-loop signals. A more detailed study of coprime factor influence on estimation accuracy was outside the scope of this paper and left for future work.

Further experiments involved increasing the flow speed and estimating the unstable plant from $\Delta$ measured at speeds within the stable range of the nominal flutter control system. At each flow speed selected for an estimate of $\Delta$, a state-space model of the unstable plant was formed and the poles of this model were extracted by calculating the eigenvalues of the $A$ matrix. One unstable pair of poles, corresponding to the flutter mode, were found at each flow speed. Table 1 lists the flow speed and the natural frequency and damping ratio of the unstable pole pair. Note that this follows the convention in structural dynamics in which a complex eigenvalue $\lambda$ is represented as

$$
\lambda=-\zeta \omega_{n} \pm j \sqrt{\zeta^{2}-1}
$$

where a negative damping ratio $\zeta<0$ indicates an unstable pole.

\section{Results of Controller Redesign}

The plant model estimated at $U_{\infty}=28 \mathrm{~m} / \mathrm{s}$ was used to redesign the flutter controller with the goal of increasing the flutter boundary beyond the capability of the nominal design. Initial designs structured $K_{2}$ as a second-order filter in the manner of $K_{1}$, but analysis showed that stabilizing the plant came at the cost of an unacceptably small low-frequency gain margin. This was attributed to the high dc gain of the plant at high flow speed. To recover the stability robustness of the nominal closed-loop system, a pair of lowfrequency complex conjugate zeros was added to $K_{2}$ to reduce its dc gain. The Evans root-locus method, in which the closed-loop poles are plotted in the complex plane as a function of a controller parameter [18], was used to select the remaining parameters of $K_{2}$. The goal of this design procedure was to achieve maximum damping of the dominant closed-loop poles, while keeping the controller order as low as possible. Good performance was achieved with a thirdorder controller of the form

$$
K_{2}(s)=\frac{g_{2}\left(s^{2}+2 \zeta_{z} \omega_{z} s+\omega_{z}^{2}\right)}{\left(s^{2}+2 \zeta_{2} \omega_{2} s+\omega_{2}^{2}\right)\left(s+\omega_{3}\right)}
$$

Figure $\underline{8}$ shows the Evans root-locus plot obtained by varying the gain $g_{2}$ while keeping the other parameters of $K_{2}$ constant at their final design values. Squares mark the design pole locations and circles mark the closed-loop zeros. The redesigned controller had the 

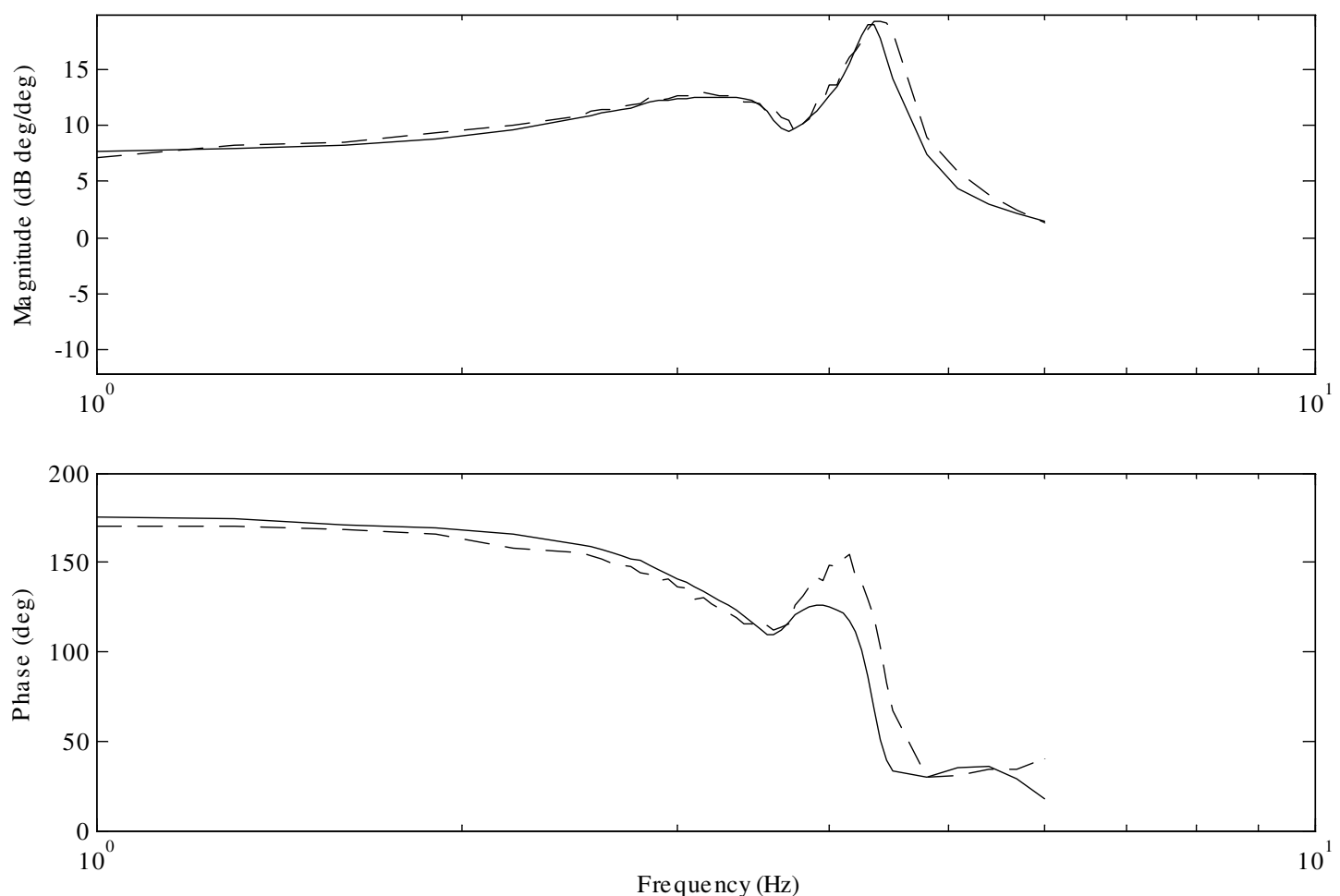

Fig. 7 Experimental frequency response of commanded flap angle to pitch angle at subcritical $U_{\infty}=22.5 \mathrm{~m} / \mathrm{s}$; measured (dashed), estimated from $\Delta$ and coprime factors (solid).

following parameter values: $g_{2}=40.1, \zeta_{z}=0.03, \omega_{z}=0.31$, $\zeta_{2}=0.3, \omega_{2}=25.1$, and $\omega_{3}=126.3$.

The redesigned controller was tested at flow speeds above those reached during the nominal controller testing. Figure 9 shows the experimental closed-loop damping ratios for both the nominal and redesigned controllers plotted versus several flow speeds. The openloop damping ratios are also shown for comparison. For the flow speeds at which the airfoil was stable, damping ratios were extracted from state-space models identified from the flap angle to the pitch angle frequency response at specific speeds. These data points are denoted by asterisks (open loop), triangles (nominal controller, closed loop), and circles (redesigned controller, closed loop). The solid lines represent cubic polynomials fit to the data. One data point from the nominal controller testing and two data points from the redesigned controller testing deviated from their respective trends, which was attributed to numerical sensitivity in the calculation of $\Delta$ and in the frequency-domain identification technique. The data points for the redesigned controller stop at $32 \mathrm{~m} / \mathrm{s}$ because a physical limitation of the test fixture prevented plant identification beyond this speed. This was due to the fact that the swept-sine flap excitation caused the airfoil plunge linkage to reach its displacement limit and initiate a potentially damaging limit cycle oscillation. Perturbing the airfoil manually to calculate the damping ratio from the resulting decaying response also proved difficult, therefore no further identification or controller redesign was attempted above $32 \mathrm{~m} / \mathrm{s}$. Despite this limitation, the flow speed was able to reach $35 \mathrm{~m} / \mathrm{s}$ before the airfoil went into flutter, an increase of $52 \%$ above the open-

Table 1 Flutter mode frequencies and damping ratios from estimated models

\begin{tabular}{lcc}
\hline \hline$U_{\infty}, \mathrm{m} / \mathrm{s}$ & Frequency, $\mathrm{Hz}$ & Damping ratio, $\zeta$ \\
\hline 23.5 & 4.03 & -0.000464 \\
24 & 3.96 & -0.0246 \\
25 & 3.93 & -0.04 \\
26 & 3.85 & -0.05 \\
27 & 3.80 & -0.064 \\
28 & 3.77 & -0.08 \\
\hline \hline
\end{tabular}

loop flutter boundary. For the damping data obtained with the redesigned controller in the loop, the cubic polynomial was fit such that it crossed zero damping at $35 \mathrm{~m} / \mathrm{s}$ to reflect what was observed in testing. It should also be noted that data in Fig. 9 indicate that the redesigned controller could stabilize the plant over the nominal range of flow speeds as well, suggesting that identifying the unstable plant could lead to improvements on previous controller iterations.

\section{Conclusions}

The technique of $Q$ parameterization was used to parameterize a model of the unstable flutter dynamics of an airfoil above its openloop flutter speed. The model consisted of a known, nominal plant and an unknown $Q$ parameter that captured differences between the true plant and the nominal model. Experiments were performed in a wind tunnel on a typical section airfoil with an articulated trailingedge flap. With a nominal controller stabilizing the airfoil, plant models at various flow speeds were estimated from $Q$ parameters measured in an open-loop manner from closed-loop signals. Comparing a known, subcritical plant frequency response to one obtained using $Q$-parameterized identification showed excellent magnitude agreement but a peak difference in phase of $35 \mathrm{deg}$. This error was potentially due to numerical sensitivity introduced by the choice of coprime factors in the plant and controller parameterizations and motivates future work in studying this effect.

The $Q$-parameterization technique identified the unstable airfoil dynamics accurately enough to redesign the flutter controller to stabilize at higher flow speeds than the nominal case, despite remaining uncertainties. The redesigned controller increased the boundary to $35 \mathrm{~m} / \mathrm{s}$ (a flow speed limited by a wind tunnel), an overall increase of $52 \%$ beyond the no-control flutter boundary of $23 \mathrm{~m} / \mathrm{s}$. This is compared to the nominal controller, which increased the flutter boundary $30 \%$ to $30 \mathrm{~m} / \mathrm{s}$. Furthermore, the redesigned controller was able to stabilize the airfoil over the same flow speed range as the nominal controller, which included a large range of flow speeds below the open-loop flutter boundary (although it should not be expected to work at all flow speeds.) Because they tolerated more flow speed deviation below their design point than above it, this suggests that the chosen controller structures did not have symmetric 


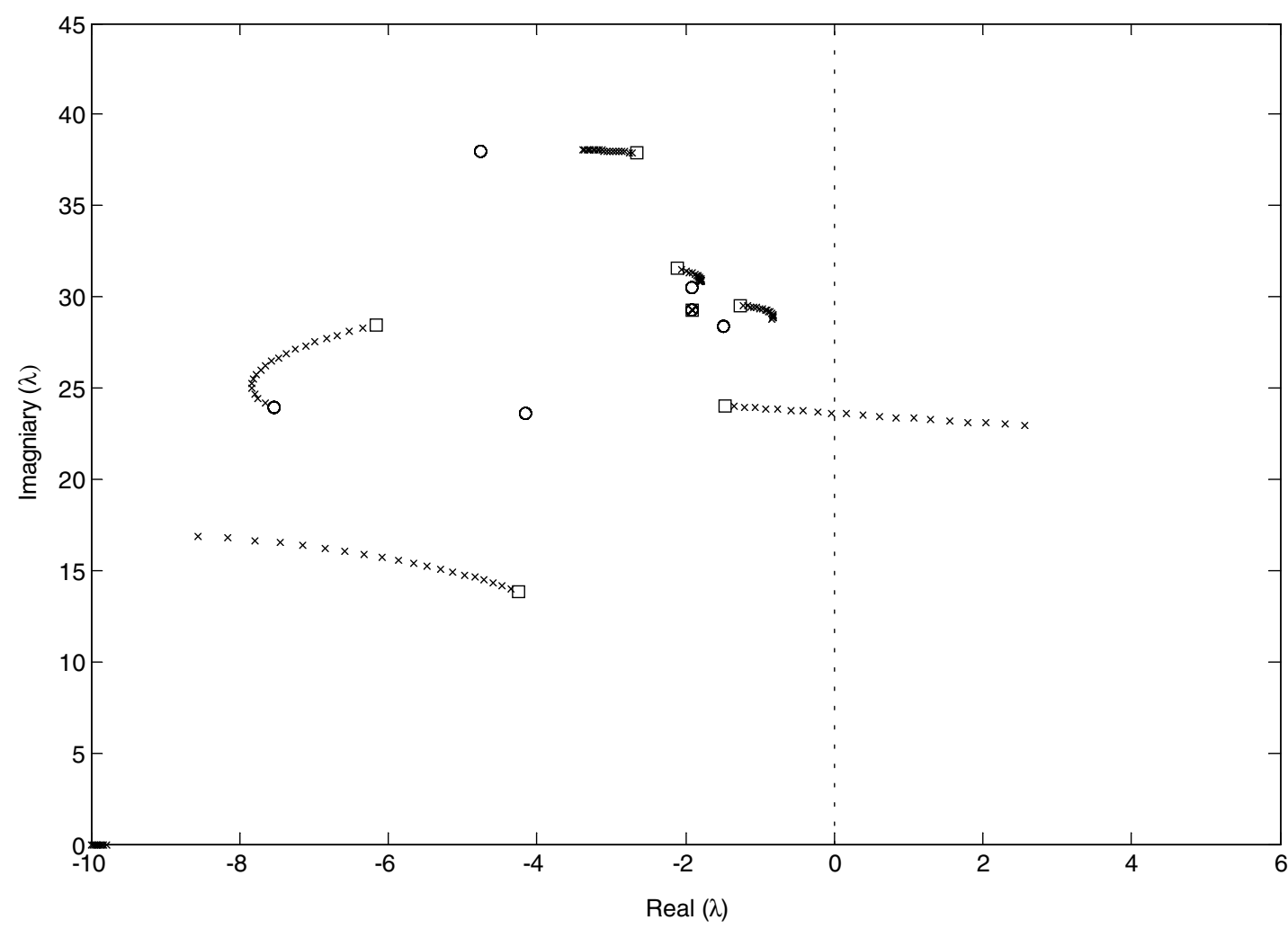

Fig. 8 Evans root-locus plot showing migration of closed-loop poles with increasing gain of $K_{2}$ coupled with $28 \mathrm{~m} / \mathrm{s}$ plant model.

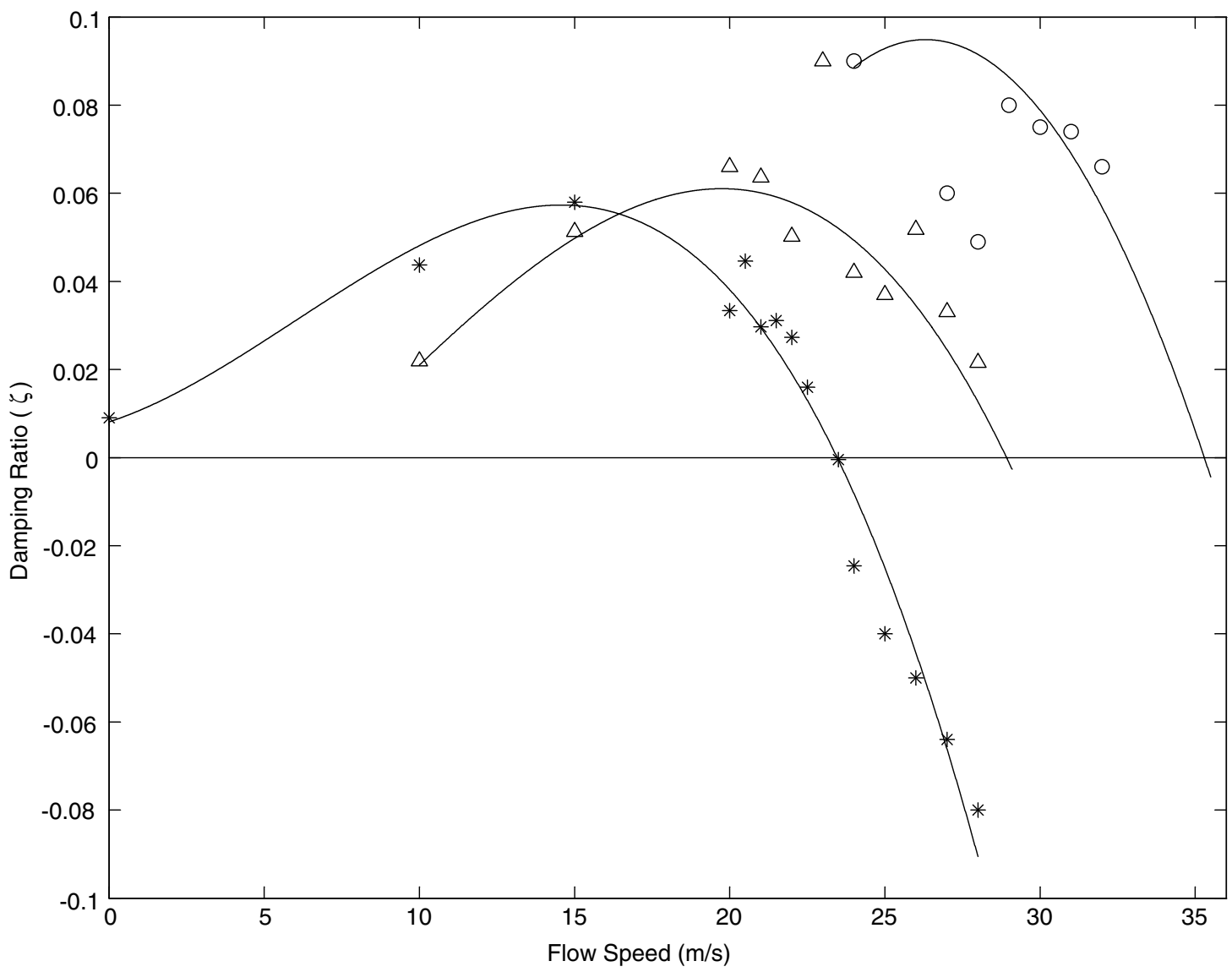

Fig. 9 Damping ratio versus flow speed.

stability robustness around the operating flow speed for which they were designed. In other words, the controllers better tolerated varying flutter mode poles that became more stable than those that became less stable. The plant at higher flow speed was more unstable and difficult to control, but the ability to accurately identify it facilitated the redesign of the better performing controller.

This work illustrated the success of the technique for the specific case of a typical section airfoil in two-dimensional flow with one 
trailing-edge flap. However, the technique is not limited to these conditions. $Q$-parameterization theory easily extends to MIMO systems and can be used to control multiple, coupled modes of flutter using multiple actuators. Future work would address issues for using the $Q$-parameterized identification technique for more complicated aircraft wings with multiple control surfaces.

\section{References}

[1] Theodorsen, T., "General Theory of Aerodynamic Instability and the Mechanism of Flutter," NACA, Tech. Rept. 496, 1935.

[2] Livne, E., "Integrated Aeroservoelastic Optimization: Status and Directions," 38th AIAA Structures, Structural Dynamics and Materials Conference, AIAA, Reston, VA, April 1997.

[3] Noll, T. E., Structural Dynamics and Aeroelasticity, Vol. 5, FlightVehicle Materials, Structures, and Dynamics-Assessment and Future Directions, ASME, New York, 1993, pp. 179-212.

[4] Perry, B. I., Cole, S. R., and Miller, G. D., "Summary of the Active Flexible Wing Program," Journal of Aircraft, Vol. 32, No. 1, 1995, pp. 10-15.

[5] Vipperman, J. S., Clark, R. L., Conner, M., and Dowell, E. H., "Experimental Active Control of a Typical Section Using a TrailingEdge Flap," Journal of Aircraft, Vol. 35, No. 2, 1998, pp. 224-229.

[6] Borglund, D., and Kuttenkeuler, J., "Active Wing Flutter Suppression Using a Trailing Edge Flap," Journal of Fluids and Structures, Vol. 16, No. 3, 2002, pp. 271-294.

[7] Ardelean, E. V., McEver, M. A., Cole, D. G., and Clark, R. L., "Flutter Suppression Using V-Stack Piezoelectric Actuator," Proceedings of the 44th AIAA/ASME/ASCE/ASC Structures, Structural Dynamics, and Materials Conference, AIAA, Reston, VA, 7-10 April 2003
[8] Roy, I. D., and Eversman, W., "Adaptive Flutter Suppression of an Unswept Wing," Journal of Aircraft, Vol. 33, No. 4, 1996, pp. 775783.

[9] Juang, J.-N., "Identification of System, Observer, and Controller From Closed-Loop Experimental Data," Journal of Aircraft, Vol. 17, No. 1, 1994, pp. 91-96.

[10] Anderson, B. D. O., "From Youla-Kucera to Identification, Adaptive and Nonlinear Control," Automatica, Vol. 34, No. 12, 1998, pp. $1485-1506$.

[11] Ljung, L., System Identification: Theory for the User, Prentice-Hall, Upper Saddle River, NJ, 1999.

[12] Hansen, F., Franklin, G., and Kosut, R., "Closed-Loop Identification via the Fractional Representation: Experimental Design," Proceedings of the American Control Conference, 1989, pp. 386-391.

[13] Tay, T. T., Moore, J. B., and Horowitz, R., "Indirect Adaptive Techniques for Fixed Controller Performance Enhancement," International Journal of Control, Vol. 50, No. 5, 1989, pp. 1941-1959.

[14] Schrama, R. J. P., "An Open-Loop Solution to the Approximate ClosedLoop Identification Problem," Proceedings of the 9th IFAC/IFORS Symposium on Identification and Systems Parameter Estimation, 1991, pp. 1602-1607.

[15] Ardelean, E. V., and Clark, R. L., "V-Stack Piezoelectric Actuator," Smart Structures and Materials, SPIE Proceedings Vol. 4333, SPIEInternational Society for Optical Engineering, Bellingham, WA, 2001, pp. 322-333.

[16] Fanson, J., and Caughey, T., "Positive Position Feedback Control for Large Space Structures," AIAA Journal, Vol. 28, No. 4, 1990, pp. 717 724.

[17] Doyle, J., Francis, B., and Tannenbaum, A., Feedback Control Theory, Macmillan, New York, 1992.

[18] Dorf, R. C., and Bishop, R. F., Modern Control Systems, AddisonWesley, Boston, MA, 1995 